\title{
Regulation gene expression of miR200c and ZEB1 positively enhances effect of tumor vaccine B16F10/GPI-IL-21 on inhibition of melanoma growth and metastasis
}

Xiaoying Wang ${ }^{1 \dagger}$, Xiangfeng He ${ }^{1,2+}$, Fengshu Zhao ${ }^{1+}$, Jing Wang ${ }^{3}$, Hongyi Zhang ${ }^{1}$, Fangfang Shi ${ }^{1,4}$, Yunxia Zhang ${ }^{1}$, Kai Cai ${ }^{1}$ and Jun Dou ${ }^{1 *}$

\begin{abstract}
Background: Genetically modified cells have been shown to be one of the most effective tumor vaccine strategies. However, in many cases, such as in melanoma, induction of a potent immune responses against the disease still remains a major challenge. Thus, novel strategies to reinforce tumor vaccine efficacy are needed. Using microRNA (miR) and Zinc-finger E-box binding homeobox (ZEB) have received much attention for potentially regulating tumor progression. To elicit a potent antitumor efficacy against melanoma, we used tumor vaccine in combination with miR200c overexpression or ZEB1 knockdown to assess the efficacy of treatment of murine melanoma.

Methods: B16F10 cell vaccine expressing interleukin 21 (IL-21) in the glycosylpho- sphatidylinositol (GPI)-anchored form (B16F10/GPI-IL-21) were developed. The vaccine was immunized into mice challenged by B16F10 cells or B16F10 cells stably transduced with lentiviral-miR200c (B16F10/miR200c) or transfected with the ZEB1-shRNA recombinant (B16F10/shZEB1) or the B16F10/GPI-IL-21 vaccine. The immune responses, tumorigenicity and lung metastasis in mice were evaluated, respectively.

Results: The vaccination with B16F10/GPI-IL-21 markedly increased the serum cytokine levels of IFN- $\gamma$, TNF-a, IL-4 and decreased TGF- $\beta$ level as well as augmented the cytotoxicity of splenocytes in immunized mice compared with control mice. In addition, the tumor vaccine B16F10/GPI-IL-21 significantly inhibited the tumor growth and reduced counts of lung metastases in mice challenged by B16F10/GPI-IL-21, B16F10/shZEB1 and B16F10/miR200c respectively compared with the control mice challenged by B16F10 cells. The efficacy mechanisms may involve in reinforcing immune responses, increasing expression of miR200c, E-cadherin and SMAD-7 and decreasing expression of TGF- $\beta$, ZEB1, Vimentin and N-cadherin in tumor tissues from the immunized mice.
\end{abstract}

Conclusions: These results indicate that the tumor vaccine B16F10/GPI-IL-21 in combination with miR200c overexpression or ZEB1 knockdown effectively inhibited melanoma growth and metastasis a murine model. Such a strategy may, therefore, be used for the clinical trials.

Keywords: Melanoma, Tumor vaccine, Interleukin -21, miR200c, Zinc-finger E-box binding homeobox 1

\footnotetext{
* Correspondence: njdoujun@seu.edu.cn

${ }^{\dagger}$ Equal contributors

'Department of Pathogenic Biology and Immunology of Medical School,

Southeast University, Nanjing 210009, China

Full list of author information is available at the end of the article
} 


\section{Introduction}

Melanoma is malignant and is one of the deadliest forms of skin cancer and its incidence is expected to rise over the next two decades. Despite significant progress in melanoma research having been made over the years, there are no effective therapies for advanced melanoma at present $[1,2]$. A growing body of literature shows that the immunotherapeutic approaches to use the immune system against cancer have focused on therapeutic vaccines that intended to specifically initiate or amplify a host response against evolving tumor cells [3-5].

Glycosylphosphatidylinositol (GPI) is a posttranslational added lipid anchor and GPI-anchored membrane cytokines have been shown to play an important role in host immune responses against tumor cells [6]. Interleukin (IL)-21 is a T cell-derived cytokine that is involved in $\mathrm{T}$ and NK cell activation in the tumor vaccine approaches. Studies have showed that IL-21 is widely applied to significantly augment antitumor immunity in multiple murine tumor models and clinical trials [7-9], and to generate considerable research interest in understanding its mode of action. Our previous study showed that administering whole tumor cell vaccine expressing IL-21 in the GPIanchored form (B16F10/GPI-IL-21) induced protective anti-melanoma immunity in a B16F10 cell transplantable mouse model. However, an anti-melanoma efficacy failed to completely induce objective tumor shrinkage because the measurable and metastatic tumors were developed in some B16F10 melanoma-bearing mice, highlighting the need for using combination and alternative strategies $[10,11]$. More recent approaches have centered on a series of molecules known as potentially regulating tumor progression such as microRNA-200c (miR200c) [12] and zinc finger E-box binding homeobox 1 (ZEB1) [13], which have provided proofs that overexpression of miR200c or knockdown of ZEB1 could repress tumor 'epithelial to mesenchymal transition' (EMT) program that activates cellular mobility, subsequent tumor metastasis [14-16].

Recent researches have demonstrated that ZEB1 has an important regulatory role on the malignant tumor progression through regulating cell cycle, apoptosis, senescence, invasion, metastasis and mesenchymal angiogenesis [12,17]. Our previous studies indicated that the disturbance of EMT-miR-200c-ZEB1 feedback loop promoted the melanoma proliferation and invasive metastasis [16], and that the miR-200c overexpression in $\mathrm{CD} 44^{+} \mathrm{CD} 133^{+} \mathrm{B} 16 \mathrm{~F} 10$ cells markedly inhibited the cell proliferation and invasion ability in vitro as well as tumorigenicity in vivo [18]. It is known that the rapid progression of primary melanomas to locally invasive and metastatic other sites is a major obstacle for therapeutic interventions of melanoma patients. On the basis of these findings, we speculate that the EMT-inducer ZEB1 may support tumor metastasis in B16F10 melanomabearing mouse model by promoting tumor cell mobility and dissemination, and that antitumor immune responses induced by tumor vaccine B16F10/GPI-IL-21 might result in tumor cell apoptosis and inhibit tumor growth but do not block the EMT of B16F10 cells or metastasis in mice. In this regard, we used the novel strategy that the tumor vaccine B16F10/GPI-IL-21 was combined with overexpression of miR200c or knockdown of ZEB1 to evaluate the synergitic efficacy of treatment of murine melanoma, and to attempt to investigate the molecular mechanisms of melanoma metastasis in the present study.

Here, we show that the tumor vaccine B16F10/GPIIL-21 in combination with potentially synergistic active therapies, such as either miR200c overexpression or ZEB1 knockdown, significantly represses tumor growth, blocks melanoma EMT program and inhibits tumor metastasis in a murine melanoma model.

\section{Materials and methods}

\section{Animals}

C57BL/6 mice between 5-6 weeks of age were obtained from the Yang Zhou University of China (license number: SCXK, Jiangsu province of China, 2007-0001). All mice were housed under the specified pathogen-free condition and the experiments were performed in compliance with the guidelines of the Animal Research Ethics Board of Southeast University.

\section{Cells}

B16F10 murine melanoma cell line is syngeneic in C57BL/ 6 mice and Human embryonic kidney (HEK) 293 T cells purchased from the Cellular Institute in Shanghai, China. B16F10 cells were cultured at $37^{\circ} \mathrm{C}$ in $5 \% \mathrm{CO}_{2}$ atmosphere in RPMI 1640 supplemented with $10 \%$ fetal bovine serum (FBS, Gibco BRL, USA) that contained $100 \mathrm{U} / \mathrm{ml}$ penicillin $\mathrm{G}$ sodium and $100 \mu \mathrm{g} / \mathrm{ml}$ streptomycin. HEK $293 \mathrm{~T}$ cells were cultured in Dulbecco's Modified Eagle Medium (DMEM, Invitrogen, NY, USA) plus 10\% FBS, $2 \mathrm{mmol} /$ $\mathrm{L}$ L-glutamine, $100 \mathrm{U} / \mathrm{ml}$ penicillin, and $100 \mu \mathrm{g} / \mathrm{ml}$ streptomycin at $37^{\circ} \mathrm{C}$ in a humidified $5 \% \mathrm{CO}_{2}$ atmosphere.

\section{Short hairpin RNA sequence design, construction of shRNA1 targeting ZEB1 gene and screen of clones stably transfected with shRNA1}

Short hairpin RNA sequence of mouse ZEB1 was designed based on the ZEB1 mRNA ID (GenBank NO. NM_011546.3) using the siDESIGN design software (Dharmacon, http://www.thermoscientificbio.com/designcenter/) and Block-iTTM RNAi Designer (Invitrogen, Grand island, NY) as well as BLAST (http://www.ncbi. nlm.nih.gov/BLAST). ShRNA sequences are as follows: ZEB1-siRNA1: sense, 5'-GATCCCCATAGAGGCTACAA GCGCTTTA-TTCAAGAGA-TAAAGCGCTTGTAGCC TCTATTTTTTGGAAA-3'; antisense, 5'-AGCTT'TTC CAAAAAATAGAGGCTACAAGCGCTTTA-TCTCTT 
GAA-TAAAGCGCTTGTAGCCTCTATGGG-3'. The square frames in nucleotide sequences contain the $9 \mathrm{nu}$ cleotide spacers, respectively. The underlined sequences contain BglII and HindIII enzyme cut sites. The primers were synthesized by Gene and Technology of China in Shanghai. A pSUPER-EGFP1 (enhanced green fluorescent protein 1) vector was used to construct recombinant pSUPER-EGFP1-ZEB1-shRNA1 as previously described [19]. A pSUPER- EGFP1-scrambled shRNA (Scramble) was used as a negative control. These recombinants were verified by the analyses of endonuclease digestion and sequencing. B16F10 cells were transfected with either the ZEB1-shRNAs or the Scramble-shRNA by using Lipofectamine $^{\text {Tx }} 2000$ reagent (Invitrogen, USA) following the manufacturer's protocol. After an antibiotic selection with $800 \mu \mathrm{g} / \mathrm{ml}$ G418 (Clontech, CA), the ZEB1-shRNA1B16F10 clones were isolated from the G418 resistant cells for each transfection pooled and expanded into cell lines and designated 'B16F10/shZEB1'. ZEB1 expression was detected by quantitative-PCR and western blotting, respectively [20].

\section{Transduction of lentivirus miR-200c and production of stable expression clones}

To generate the miR-200c expression lentivirus vector, we amplified an insert (full-length mouse miR-200c) by PCR from B16F10 cell DNA. The lentivirus miR-200c was produced from the transient transfection of the HEK293T cells with pHAGE-CMV-miR-200c-IZsGreen, psPAX2, and pMD2.G plasmid DNAs plus Lipofectamine 2000 (Invitrogen, USA) according to the manufacturer's protocol. Forty-eight hours after the cotransfection, the lentivirus- bearing supernatants were collected and passed through a $0.45-\mathrm{mm}$ filter. B16F10 cells were infected with the pHAGE-CMV-miR-200c-IzsGreen lentivirus, and were selected by the IzsGreen expression [21]. The stable expression clones were selected by limiting the dilution assay and designated'B16F10/miR200c' [22].

\section{Quantitative-PCR assay}

Quantitative-PCR analysis was performed on an ABI step one plus real-time system (Applied Biosystems). The comparative $\mathrm{Ct}(\Delta \Delta \mathrm{Ct})$ method was used to determine the expression fold change [12]. Total cellular RNA was isolated from each sample by using a Qiagen RNeasy Kit (Qiagen, Valencia, CA). One microgram of total RNA from each sample was subjected to cDNA synthesis using the Superscript III reverse transcriptase (Invitrogen). cDNAs were amplified by PCR with primers as follows: miR-200c (sense, 5' -GAAGATCTG GAGCAGG AGATCTGCCGCTTC-3; reverse, GGAA TTCAGAGCCACCCTTAACTCGG); ZEB1(sense, 5' -T GAGCACACAGGTAAGAGGCC-3'; reverse, 5'-GGC TTTTCCCCAGAGTGCA-3'); $\beta$-actin (sense, $5^{\prime}$-GC
CCTGAGGCTCTTTTCCA -3'; reverse, 5'-TTACGG ATGTCAACGTC A-3'); U6 (sense, 5'-CTCGCTTC GGCAGCACATAGG-3'; reverse, 5'-AACGCTTCAC GAA TTTGCG TAGGAG-3'); URP Universal Reverse Primer, 5' -CCGGCAGGGTCCGAGGT-3'.

\section{Western blot}

$1 \times 10^{6}$ different cells were collected and lyzed in the protein extraction buffer (Novagen, Madison, WI, USA) by following the manufacturer's protocol. $12 \%$ sodium dodecyl sulfate-polyacrylamide gel electrophoresis was performed and the proteins $(10 \mu \mathrm{g} / \mathrm{lane})$ were transferred onto a PVDF membrane blocked with $4 \%$ dry milk in Trisbuffered saline with Tween- 20 for $1 \mathrm{~h}$ at $20^{\circ} \mathrm{C}$, and then incubated with the goat anti-mouse IL-21 (I-18, Santa Cruz Biotechnology Company, Santa Cruz, CA, USA), rabbit anti-mouse ZEB1, Vimentin, E-cadherin, N-cadherin, TGFßand SMAD-7 (Bioworld Technology, Dublin, OH, USA), respectively for overnight at $4^{\circ} \mathrm{C}$. The membrane was rinsed for 5 min with an antibody wash solution for 3 times before adding goat anti-rabbit or rabbit anti-goat fluorescence secondary antibody for $1 \mathrm{~h}$ at $20^{\circ} \mathrm{C}$. Immunoreactive bands were detected by Odyssey scanning instrument (LICOR Odyssey Imaging System, USA) [16].

\section{Animal experiment}

C57BL/6 mice were initially immunized subcutaneous (s.c.) with $1 \times 10^{6}$ B16F10/GPI-IL-21 tumor vaccine inactivated with $2 \mu \mathrm{g} / \mathrm{ml}$ mitoxantrone in a mouse's right flank, and the immunization was performed three times at a week interval [16]. The B16F10/GPI-IL-21 tumor vaccine was developed as previously described [10] and stored in our Lab. About 10 days after the final immunization, the mice were randomly divided into four groups: the B16F10/wild type (WT) group; B16F10/shZEB1 group; B16F10/miR200 group and B16F10/GPI-IL-21 group. Finally, all mice were challenged s.c. with $2 \times 10^{5}$ abovementioned different B16F10 cells. Six mice/group were used in the study, and the experiment was repeated twice. Tumor growth was monitored every three day by measuring two perpendicular tumor diameters using calipers, and then the counts of lung metastases [15] were also examined after mice were sacrificed.

\section{Immunosorbent assay}

Immunosorbent assay (ELISA) for detecting IFN- $\gamma$, TNF$\alpha$, IL- 4 and TGF- $\beta$ was performed by following the Kit's protocol (eBioscience, CA). The Kit is suitable for detection of samples including cell culture supernatant and serum, and the sensitivity of Kit is $5.3 \mathrm{pg} / \mathrm{mL}[23,24]$.

\section{Assay of splenocyte cytotoxicity}

Ten days after the final immunization, $5 \times 10^{6}$ splenocytes were harvested from the mice immunized with the 
inactivated tumor vaccine B16F10/GPI-IL-21, and were used for effector cells that were labeled with $0.5 \mathrm{mM} \mathrm{5-}$ (and 6)-carboxy-fluorescein diacetate succinimidyl ester $\left(\mathrm{CFSE} ; 20 \mu \mathrm{g} / \mathrm{ml}\right.$ ) at $37^{\circ} \mathrm{C}$ for $15 \mathrm{~min}$. The labeled splenocytes were washed twice in PBS containing 5\% FBS to sequester any free CFSE. The CFSE-labeled effector cells were seeded with a constant number of B16F10 target cells in a 96-well plate at 25:1 ratios of effector cells to target cells (E:T). The cytotoxicity assay was performed in triplicate. Flow cytometric CFSE/7-AAD cytotoxicity assay was analyzed by Flow Cytometry (FCM, BD company, USA) [11,25].

\section{Immunohistochemistry}

$4 \mu \mathrm{m}$-thin formalin fixed and paraffin-embedded slides were incubated with the rabbit anti-mouse ZEB1, Vimentin, E-cadherin, N-cadherin, TGF-ßand SMAD-7 (Bioworld Technology, Dublin, OH, USA), respectively overnight at $4^{\circ} \mathrm{C}$. Antibody concentration was 1:1000. The samples were then labeled with horseradish peroxidase-conjugated streptavidin (Invitrogen) and the chromogenic reaction that was developed using Liquid DAB Substrate Pack according to the manufacturer's instructions. The stained cells from random and non-overlapping fields were counted under a magnification of $\times 400[15,26]$.

\section{Statistical analysis}

Values of interest was presented as the mean plus or minus standard deviation. Statistical comparisons were performed using the Student's $t$-test and Fisher/Chisquare test methods. A $P$ value of $<0.05$ was considered statistically significant.

\section{Results}

\section{Screen of transfected and transduced clones and} identification of expression of IL-21, miR-200C and ZEB1 in a different B16F10 cells

To develop B16F10/miR200c and B16F10/shZEB1, we first constructed the miR-200c lentivirus vector and shZEB1 recombinant and then transduced and transfected them into B16F10 cells, respectively, and finally the clones stably transduced with miR-200c lentivirus vector or transfected with shRNA1 recombinant were screened. Figures 1A-D show the B16F10 cells and the B16F10/IL-21-GPI cells were observed under a light microscope (left-panels) and under a fluorescence microscope(right-panels), in which the fluorescence is displayed on the surface of B16F10/IL21-GPI cells, and the nucleus with DAPI (Figure 1D), but no green florescence on the surface of B16F10 cells in (Figure 1C). This result suggested IL-21 was expressed on the surface of B16F10/IL-21-GPI cells, which was further confirmed by the result of western blot. IL-21 band was found in B16F10/IL-21-GPI cells isolated from the G418 resistant cells as is shown in Figure 1E.
The B16F10/shZEB1 cell clones (Figure 1F) and the B16F10/miR200c cell clones (Figure 1J) were selected by limiting dilution assay, and were observed under a light microscope, and the same clones were observed under a fluorescence microscope (Figure $1 \mathrm{G}$ and $\mathrm{K}$ ). The results suggested that the clones stably transfected with shRNA1 or stably infected with lentivirus miR-200c were successfully isolated from the B16F10 cells. After the endogenous expression of miR-200c or ZEB1 in the B16F10 cells was identified by RT-PCR (data not shown), we analyzed the expression of miR-200c and ZEB1 in the transfected and transduced B16F10 cells. It was found that the expression of ZEB1 was higher in the B16F10 wild type (WT) cells than in B16F10/shZEB1 cells, whereas the miR-200c expression was obviously increased in B16F10/ZEB1 cells compared with B16F10 WT cells (Figure $1 \mathrm{H}$ and I). Figure $1 \mathrm{~L}$ indicates miR-200c expression is lower in the B16F10 WT cells than in B16F10/miR-200c cells, whereas the expression of ZEB1 was higher in the B16F10 WT cells than in B16F10/ miR-200c cells (Figure 1L and M). These molecular expression differences were statistically significant as are shown in Figure 1.

\section{Changes of serum cytokine levels and cytotoxicity of splenocytes in the mice immunized with the tumor vaccine B16F10/GPI-IL-21}

To test the immune efficacy of the tumor vaccine B16F10/GPI-IL-21 we first detected serum cytokine levels in the immunized mice. Figures 2A-D indicate that compared with mice immunized with the B16F10 cells, the serum cytokine levels of IFN- $\gamma$, TNF- $\alpha$ and IL- 4 were significantly increased after the mice were immunized with the tumor vaccine B16F10/GPI-IL-21, whereas the serum TGF- $\beta$ level was markedly decreased, which was statistically significant (A-D). Next we analyzed the cellular immune response in the immunized mice. It was found that the cytotoxicity of splenocytes to B16F10 target cells at 25:1 ratio was notably enhanced $(61 \pm 2.6 \%)$ in mice immunized with the tumor vaccine B16F10/ GPI-IL-21 in contrast to mice immunized with the B16F10 cells $(23 \pm 2.8 \%)$, and the difference was statistically significant $(P=0.0003)$. These data suggested that the tumor vaccine B16F10/GPI-IL-21 induced the mice to generate a strong immune responses.

\section{Tumor vaccine B16F10/GPI-IL-21 in combination with miR200c overexpression or ZEB1 knockdown reduced melanoma growth and metastasis}

To augment therapeutic B16F10 melanoma efficacy by tumor vaccine B16F10/ GPI-IL-21 in C57BL/6 mice, we adopted a novel strategy that the tumor vaccine B16F10/ GPI-IL-21 were combined with miR200c overexpression or ZEB1 knockdown in B16F10 cells in current antitumor experiment. Figure 3A gives the images of mice challenged 

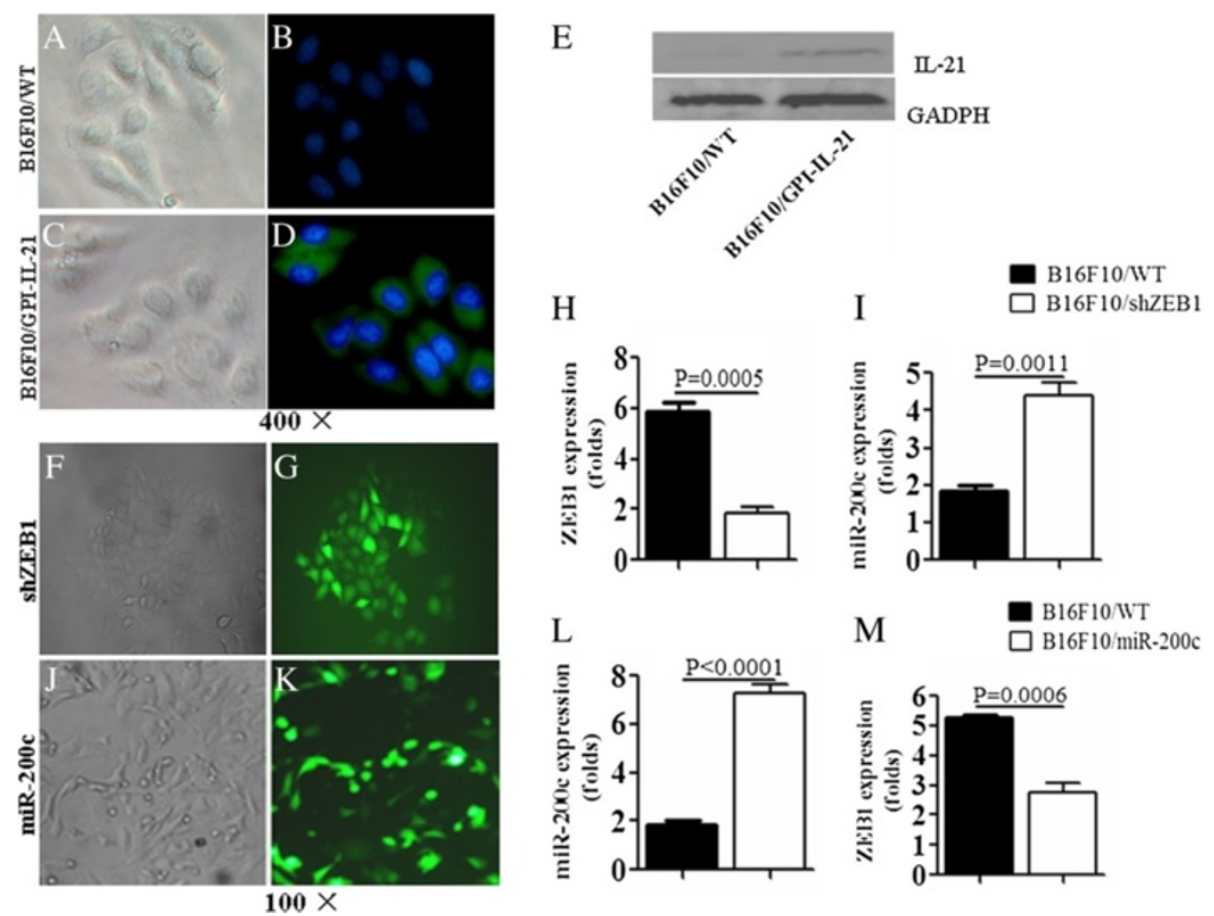

Figure 1 Observation of the different clones and detection of expression of IL-21, miR-200c and ZEB1 in B16F10 cells. (A-D, F, G, J, K) Morphological photos of B16F10 wild type (WT) clones, B16F10/GPI-IL-21 clones, B16F10/shZEB1 clones and B16F10/miR200c clones in order. The clones in left side were observed under a light microscrope, and the clones in right side were observed under a fluorescence microscrope. Magnification $400 \times(\mathbf{A}-\mathbf{D})$, and magnification $100 \times(\mathbf{F}, \mathbf{G}, \mathbf{J}, \mathbf{K})$. (D) The fluorescence on the surface of B16F10/GPI-IL-21 cells, but no fluorescence on the surface of B16F10 WT cells (B). (E) The positive band IL-21 in lane 2 in B16F10/GPI-IL-21 cells detected by western blot. (H, I) The expression of ZEB1 RNA and miR200c in shZEB1 stably transfected B16F10/shZEB1 cells and B16F10 WT cells detected by qRT-PCR. (L, M) The expression of miR200c and ZEB1 in lentivirus miR-200c stably transduced B16F10/miR200c cells and B16F10 WT cells detected by qRT-PCR.

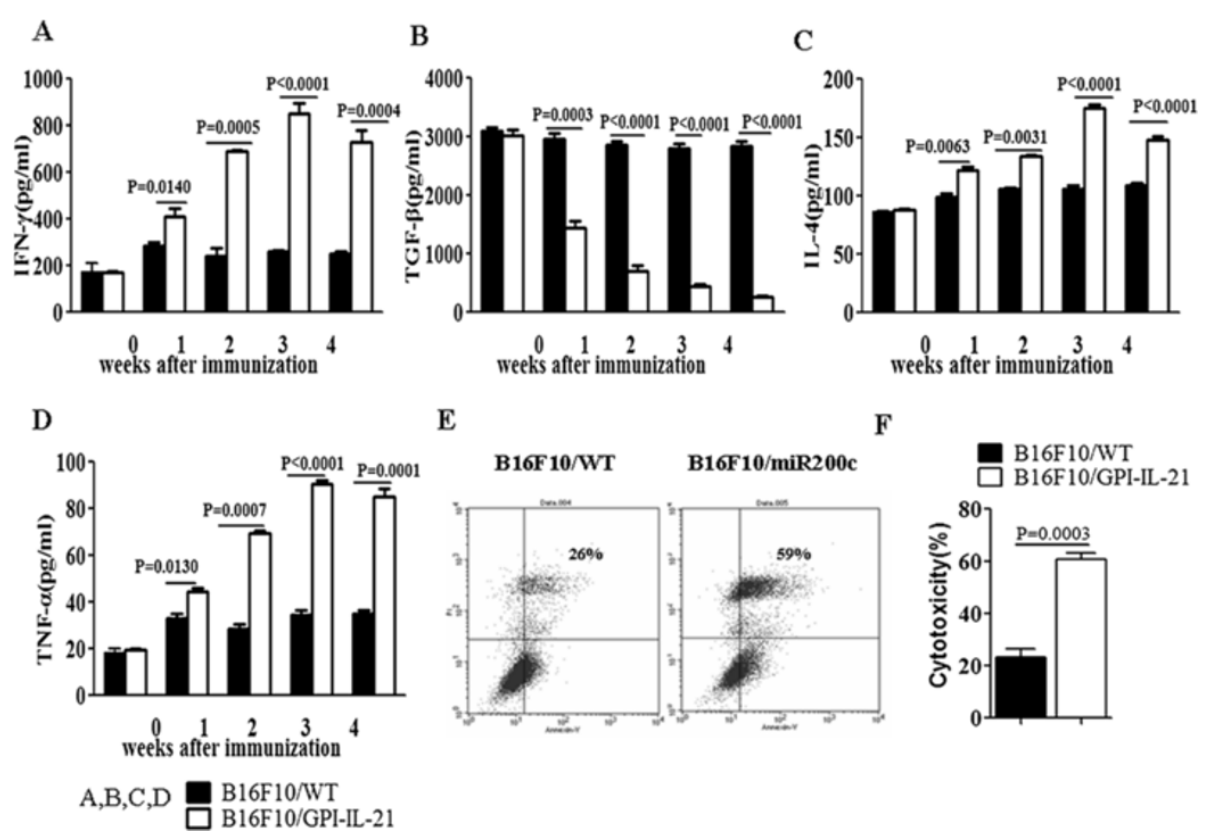

Figure 2 Tumor vaccine B16F10/GPI-IL-21 induced immune responses in mice. (A-D) Serum cytokine levels of IFN- $\gamma$, TNF- $a$, IL-4 and TGF- $\beta$ were respectively detected by ELIAS in the B16F10/GPI-IL-21 vaccination of mice. (E) The splenocyte cytotoxicity to B16F10 cells at 25:1 ratio was analyzed by FCM in the immunized mice. (F) The analysis of splenocyte cytotoxicity. 

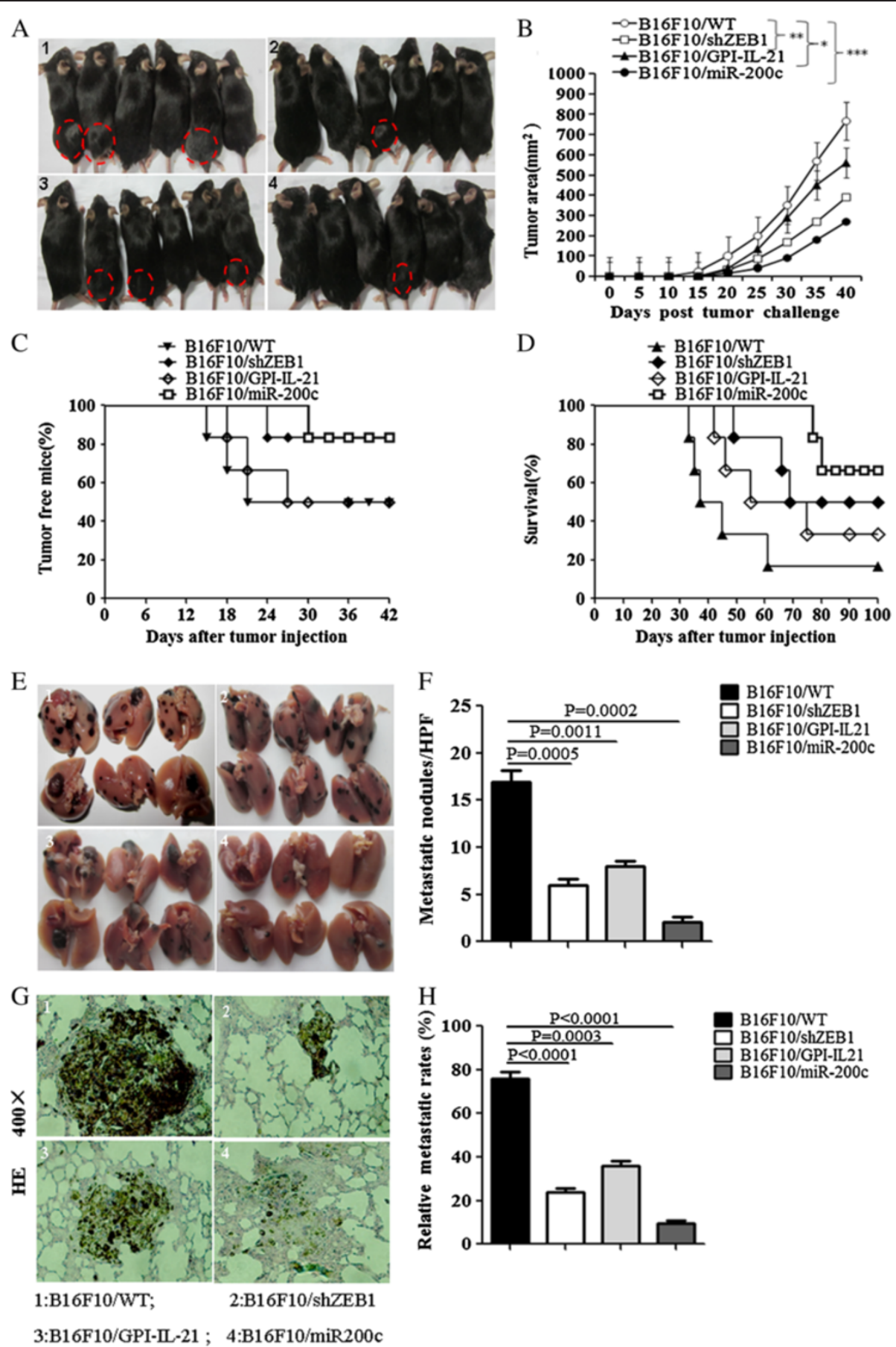

Figure 3 B16F10/GPI-IL-21 vaccination of C57BL/6 mice in combination with either miR200c overexpression or ZEB1 knockdown against B16F10 melanoma. (A) Images of the B16F10/ GPI-IL-21 vaccine immunized mice at 40 days after the mice were challenged by the different B16F10 cells. (B-D) Tumor volumes, tumor free mice and melanoma bearing mouse survival. (E) Images of metastasis nodules in murine lung. (F) The statistical analysis of metastatic tumor nodules. (G) The pathological changes in murine lung from the immunized mice that were challenged by the different B16F10 cells. (H) The statistical analysis of relative lung metastatic rates.

by the different treated B16F10 cells at 40 days after the mice were immunized with the tumor vaccine B16F10/ GPI-IL-21 three times. We found that 3 of the 6 immunized mice developed tumors on Day 15, Day 18 and Day
21 , respectively after the 6 mice were challenged by the B16F10 cells. The immunized mice challenged by the inactivated B16F10/GPI-IL-21tumor vaccine also developed tumors on Day 18, Day 21 and Day 27, respectively 
(3/6), but the tumor volumes were smaller than that of mice challenged by the B16F10 cells as is shown in Figure 3B.

Compared with the immunized mice that were challenged by the B16F10 cells, only 1 out of the 6 mice developed tumor after the mice were challenged by the B16F10/shZEB1 cells, and the measurable tumors were not detected in other 5 mice until 60 days into the observation, but the most powerful antimelanoma efficacy was found in the immunized mice that were challenged by the B16F10/miR200c cells, which was reflected in 1 out of the 6 mice developing the smaller tumor, the longer survival time, the lower tumor metastasis counts and the weaker pathological changes in murine lung than those of other mice that were shown in Figure 3. The results from the tumor area and tumor metastasis counts in lungs suggested that the synergism antitumor efficacy was found in mice immunized with the tumor vaccine B16F10/GPI-IL-21 in combination with either overexpression of miR200c or knockdown of ZEB1 in B16F10 cells.

\section{EMT-related molecular expression in tumor tissues in mice}

To analyze the mechanisms of the overexpression of miR200c or knockdown of ZEB1 for reinforcing the antimelanoma efficacy of the tumor vaccine B16F10/GPI-IL-
21, we detected the EMT-related molecular expression in tumor tissues from the immunized mice. It is known that EMT is a process associated with many factors $[12,17]$, of which TGF- $\beta$, Vimentin, ZEB-1, SMAD-7, Ecadherin and $\mathrm{N}$-cadherin molecules are closely associated with the typical phenotype change of EMT in the process of tumor cell growth [22,27]. The representative results in Figure 4A showed these molecular expression in tumor tissues detected by western blotting.

It was found that the expression of TGF- $\beta$, Vimentin, ZEB-1 and N-Cad was significantly decreased in tumor tissues from the B16F10/GPI-IL-21 vaccination of mice challenged by the B16F10/miR200c cells compared with other groups, whereas the expression of SMAD-7 and E-cadherin was significantly increased in tumor tissues, and the differences were statistically significant as are shown in Figures 4B-C. Consistent with the results of western blotting, the immunohistochemical analysis of tumor tissue sections showed that the molecular expression of TGF- $\beta$, Vimentin, ZEB-1 and N-cadherin was also reduced in tumor tissues, and that the SMAD-7 and E-cadherin expression was remarkably increased in the B16F10/GPI-IL-21 vaccination of mice that were then challenged by the B16F10/miR200c cells compared with B16F10/WT cells (Figure 5). From these results, we concluded that the B16F10/GPI-IL-21 vaccination of
A

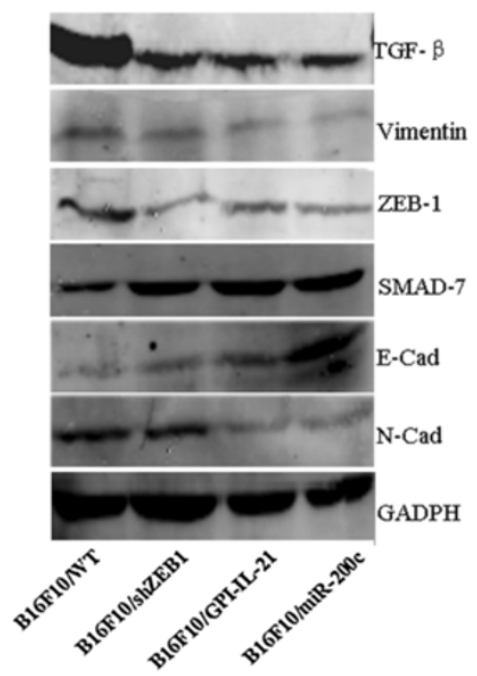

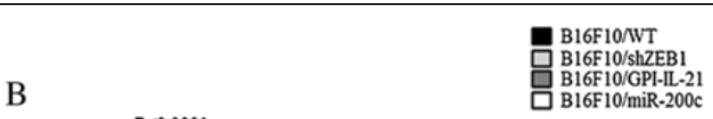
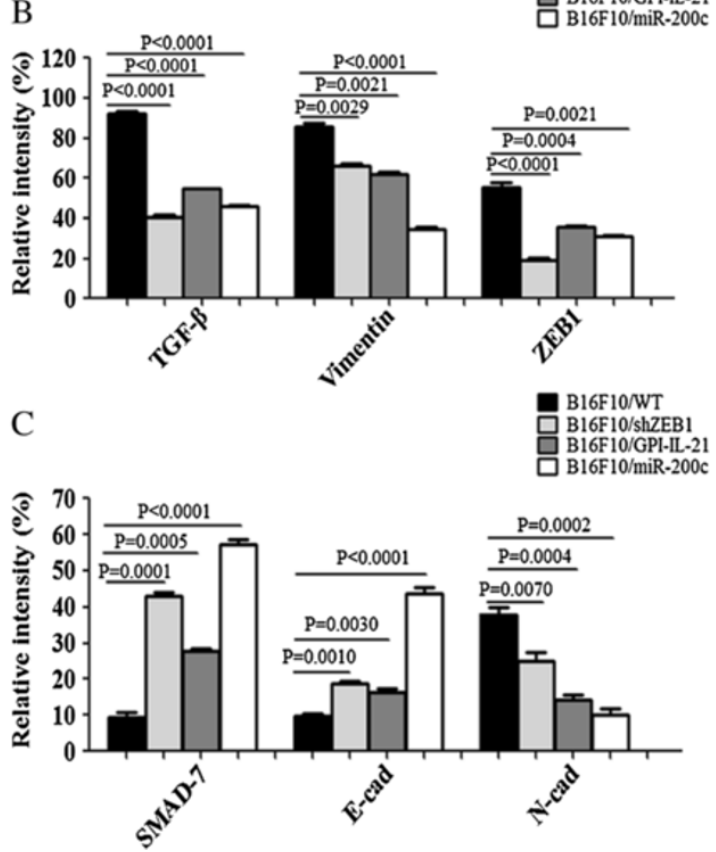

Figure 4 EMT-related molecular expression detected by western blot. (A) The EMT-related molecular expression was examined by western blot in B16F10 WT cells, B16F10/shZEB1 cells, B16F10/GPI-IL-21cells, and B16F10/miR200c cells, respectively. (B, C) The statistical analysis of relative intensity of molecular expression and the detection of the band intensities are all within the same dynamic range. 


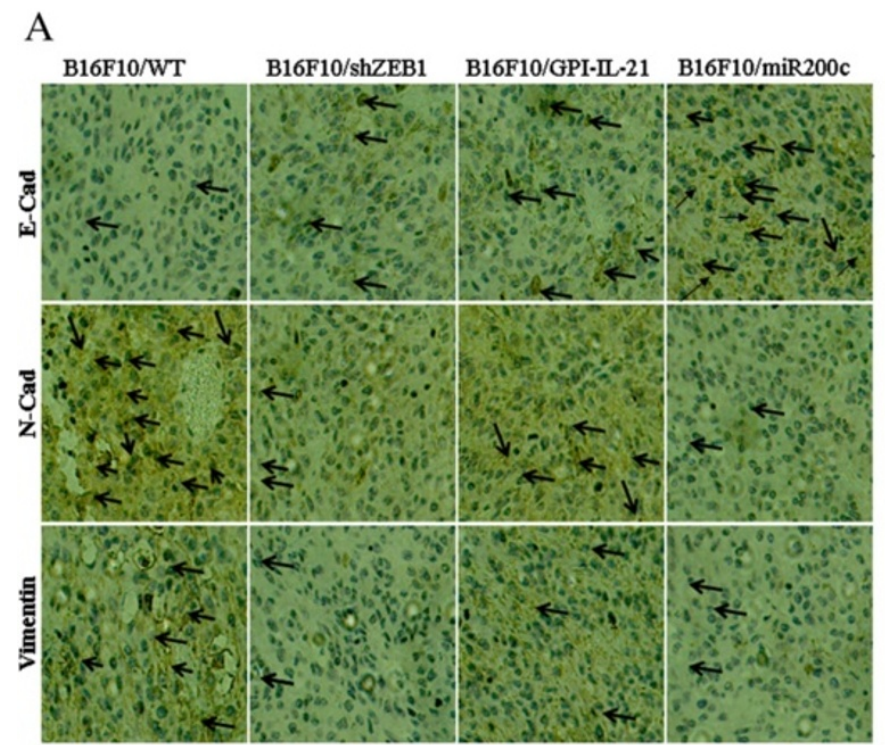

IHC $400 \times$

B
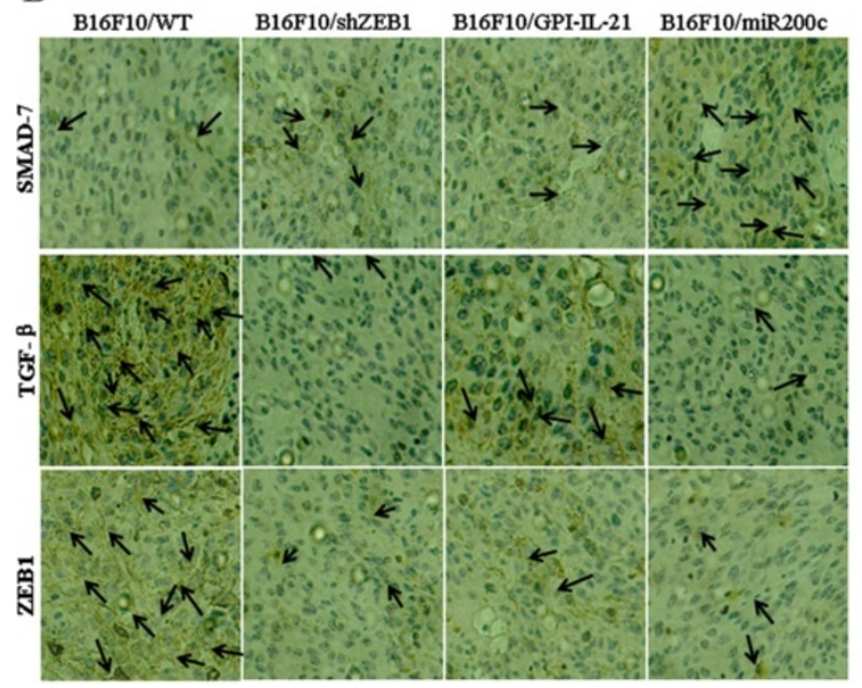

IHC $400 \times$
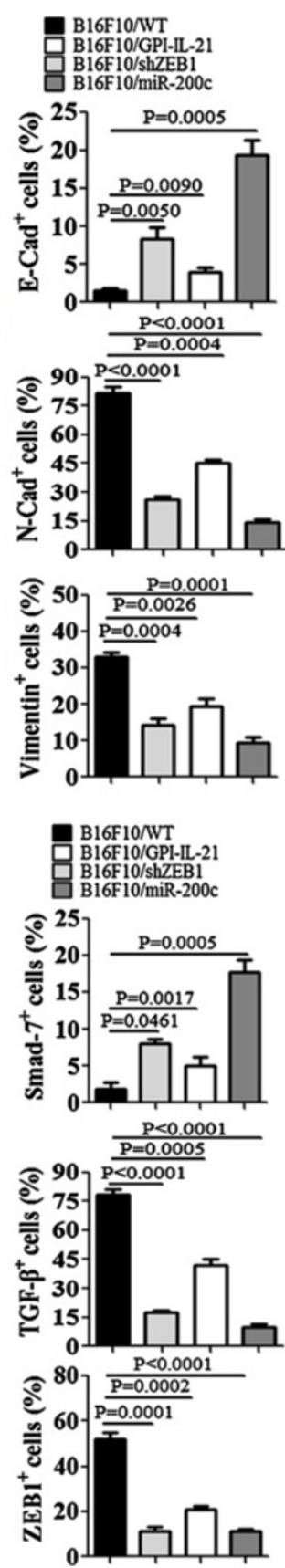

Figure 5 EMT-related molecular changes detected by immunohistochemistry assay. (A, B) The EMT-related molecular expression was examined by immunohistochemistry assay in B16F10 WT cells, B16F10/shZEB1 cells, B16F10/GPI-IL-21cells, and B16F10/miR200c cells, respectively. The statistical analysis of relative intensity of molecular expression were shown in histograms.

mice could result in the changes of EMT-related molecular expression in tumor tissues from the mice challenged by the B16F10/miR200c cells or the B16F10/ shZEB1 cells that increased miR200c expression or decreased ZEB1 expression. The high expression of SMAD-7 and E-cadherin, accompanied with low expression of TGF- $\beta$, Vimentin, ZEB- 1 and N-cadherin, may inhibit the EMT of B16F10 cells.

\section{Discussion}

In the present study, our goal was to use a tumor vaccine B16F10/GPI-IL-21 in combination with regulation expression of miR200c and ZEB1 against melanoma, an aggressive skin cancer that there is no cure in advanced stages until now. We first selected the B16F10/GPI-IL21 vaccine as an immune agonist to induce the mice to generate a strong immune responses such as increase of 
the serum cytokine levels of IFN- $\gamma$, TNF- $\alpha$, IL-4, decrease of TGF- $\beta$ level and enhancement of the splenocyte cytotoxic activity in the immunized mice. Since the malignant melanocytes can spread to distant locations and cause metastasis this process is involved in the EMT of melanoma in which miR200c and ZEB1 may be downregulation or upregulation. Therefore, we next combined the tumor vaccine B16F10/GPI-IL-21 with miR200c overexpression or ZEB1 knockdown in B16F10 cells to test the novel strategy for optimizing the therapeutic management of melanoma growth and metastasis in mice.

In an experimental model of melanoma growth and metastases, we found the tumor growth was significantly inhibited in the B16F10/GPI-IL-21 vaccination of mice that were then challenged by the B16F10/miR200c cells, which was reflected in weaker tumorigenicity, smaller tumor volumes, lower lung metastases and longer survival in melanoma bearing mice than those of mice that were challenged by the B16F10 cells (Figure 3). Even though, the B16F10/GPI-IL-21 vaccination of mice challenged by the B16F10/shZEB1 cells or B16F10/GPI-IL21 cells also indicated antitumor efficacy this efficacy was remarkably found in B16F10/miR200c cell group.

To address the functional significance of the miR200c overexpression or ZEB1 knockdown in B16F10 cells, we tested the EMT-related molecular expression in tumor tissues. It is known that the increased expression of TGF- $\beta$, Vimentin, ZEB-1and N-cadherin could promote EMT progression of tumor cells [27-30], whereas the decreased expression of SMAD-7 and E-cadherin could inhibit the EMT progression $[31,32]$. It has been observed that the expression of TGF- $\beta$, Vimentin, ZEB- 1 and $N$-cadherin detected by western blot was markedly decreased, accompanied with increased expression of SMAD-7 and Ecadherin in the B16F10/GPI-IL-21 vaccination of mice challenged by the B16F10/miR200c cells. Consistent with the western blot results, the expression of TGF- $\beta$, Vimentin, ZEB-1 and N-cadherin detected by immunohistochemistry assay was also remarkably decreased, whereas the expression of SMAD-7 and E-cadherin was notably increased in the tumor tissues. On the basis of these findings, we speculated that enforced miR-200c expression in melanoma B16F10 cells profoundly impairs cell tumorigenicity and phenotype change of EMT along with significantly decreased expression of TGF- $\beta$, Vimentin, ZEB-1and N-cadherin. Since the regulation of ZEB1 expression is also associated with the cell's EMT, the tumor metastases and change of molecular expression abovementioned were investigated in vivo experiment as well. We found that ZEB1 knockdown may correspond to reduced tumorigenicity and EMT change of B16F10 cells as well as melanoma metastases in vivo mouse model.

Several molecules, for example, IFN-gamma, SMAD-7 and E-cadherin confer inhibition of EMT to B16F10 cells
$[28,29,33,34]$. It was reported that IFN- $\gamma$ and TGF- $\beta$ have opposite effects on diverse cellular functions. IFN- $\gamma$ inhibits the TGF- $\beta$-induced phosphorylation of SMAD-3 and the accumulation of SMAD- 3 in the nucleus, and the activation of TGF- $\beta$-responsive genes [33-35]. Based on the experiment results that the serum IFN- $\gamma$ was increased, and TGF- $\beta$ was decreased as well as SMAD-7 expression was increased in tumor tissues from the immunized mice, we presumed that IFN- $\gamma$ may specifically inhibit an early step in the TGF- $\beta$-induced activation of SMAD-3 through a receptor serine kinase that phosphorylates and activates the transcription factors SMAD-2 and SMAD-3. Since IFN- $\gamma$ also induces the expression of SMAD-7, an antagonistic SMAD, which prevents the interaction of SMAD-3 with the TGF- $\beta$ receptor [33], therefore, we guess the increased SMAD-7 in tumor cells may bind to the TGF- $\beta$-receptor complex, preventing its interaction with, and phosphorylation of SMAD-3 [36,37], which may result in inhibition of melanoma growth and metastasis in mice. In addition, miR200c overexpression or ZEB1 knockdown is most likely to suppress tumor malignancy by enhancing E-cadherin expression (Figures 4 and 5A) and/or by inhibiting signals that suppress E-cadherin function.

\section{Conclusions}

Our data represents the first attempt to improve the tumor vaccine B16F10/GPI-IL-21 efficacy in combination with miR200c overexpression or ZEB1 knockdown in B16F10 cells. This efficacy resulted in inhibition of melanoma growth and metastasis in melanoma bearing mouse model. The tumor vaccine B16F10/GPI-IL-21 improves IFN- $\gamma$ secretion and induces antitumor immune responses. Regulation expression of miR200c and ZEB1 in B16F10 cells positively led to expression changes of TGF- $\beta$, Vimentin, ZEB-1, N-cadherin, SMAD-7 and Ecadherin in tumor tissues from the B16F10/GPI-IL-21 vaccination of mice, which are correlated with progression of EMT of B16F10 cells as well as influence of melanoma growth and metastasis in mice. These findings encourage the exploitation of these combined strategies in clinic trials.

\section{Abbreviations \\ EMT: Epithelial to mesenchymal transition; GPI: Glycosylpho sphatidylinositol; miR200c: microRNA-200c; ZEB1: Zinc-finger E-box binding homeobox 1; \\ CFSE: Carboxyfluoroscein diacetate succinimidyl ester; FACS: \\ Fluorescence-assisted cell; ELISPOT: Enzyme-linked immunospot; \\ TGF- $\beta$ : Transforming growth factor- $\beta$; IFN- $\gamma$ : Interferon $\gamma ;$ TNF-a: Tumor \\ necrosis factor $\alpha$; IL-4: Interleukin 4}

\section{Competing interests}

The authors declared that they have no competing interests.

\section{Authors' contributions}

$\mathrm{XW}, \mathrm{FZ}$ and $\mathrm{XH}$ carried out the experiments described in the manuscripts, developed the technique described in the manuscript, and participated in the writing of the manuscript. JW, HZ, FS, YZ, KC and JS participated in most 
of the experiments. JD and FZ contributed to the design of the experiments and contributed to the writing of the manuscript. All authors have read and approved the final manuscript.

\section{Authors' information}

Xiaoying Wang, Xiangfeng He and Fengshu Zhao are co-authors.

\section{Acknowledgements}

We thank Dengyu Cheng, Wenhu Cao, and Yurong Liu for their help with short hairpin RNA sequence design and transduction of lentivirus miR-200c.

\section{Financial support}

This work was supported by the National Natural Science Foundation of China (No. 81071769, No. 81202372), and in part by the 973 National Nature Science Foundation of People's Republic of China (2011CB933500).

\section{Author details}

'Department of Pathogenic Biology and Immunology of Medical School, Southeast University, Nanjing 210009, China. ${ }^{2}$ Department of Medical Oncology, Affiliated Tumor Hospital of Nantong University, Nantong 226361, China. ${ }^{3}$ Department of Gynecology \& Obstetrics, Zhongda Hospital, Medical School, Southeast University, Nanjing 210009, China. ${ }^{4}$ Department of Oncology, Zhongda Hospital, Southeast University, Nanjing 210009, China.

Received: 19 January 2014 Accepted: 4 March 2014

Published: 14 March 2014

\section{References}

1. Quintana E, Shackleton M, Foster HR, Fullen DR, Sabel MS, Morrison SJ: Phenotypic heterogeneity among tumorigenic melanoma cells from patients that is reversible and not hierarchically organized. Cancer Cell 2010, 18:510-523.

2. Romano S, Staibano S, Greco A, Brunetti A, Nappo G, llardi G, Martinelli R, Sorrentino A, Di Pace A, Mascolo M, Bisogni R, Scalvenzi M, Alfano B, Romano MF: FK506 binding protein 51 positively regulates melanoma stemness and metastatic potential. Cell Death Dis 2013, 4:e578.

3. Farolfi A, Ridolfi L, Guidoboni M, Nicoletti SV, Piciucchi S, Valmorri L, Costantini M, Scarpi E, Amadori D, Ridolfi R: Ipilimumab in advanced melanoma: reports of long-lasting responses. Melanoma Res 2012, 22:263-270.

4. Drake CG, Lipson EJ, Brahmer JR: Breathing new life into immunotherapy: review of melanoma, lung and kidney cancer. Nat Rev Clin Oncol 2014, 11:24-37.

5. Kwek SS, Cha E, Fong L: Unmasking the immune recognition of prostate cancer with CTLA4 blockade. Nat Rev Cancer 2012, 12:289-297.

6. Naghibalhossaini F, Yoder AD, Tobi M, Stammers CP: Evolution of a tumorigenic property conferred by glycophosphatidyl-inositolmembrane anchors of carcinoembryonic antigen gene family members during the primate radiation. Mol Biol Cell 2007, 18:1366-1374.

7. Davis ID, Brady B, Kefford RF, Millward M, Cebon J, Skrumsager BK, Mouritzen U, Hansen LT, Skak K, Lundsgaard D, Frederiksen KS, Kristjansen PE, McArthur G: Clinical and biological efficacy of recombinant human interleukin-21 in patients with stage IV malignant melanoma without prior treatment: a phase lla trial. Clin Cancer Res 2009, 15:2123-2129.

8. Davis JM, Harris MP, Davidson NE, Kobrin BJ, Jaffee EM, Emens LA: Costeffective manufacture of an allogeneic GM-CSF-secreting breast tumor vaccine in an academic cGMP facility. Cytotherapy 2005, 7:46-56.

9. Shu S, Cochran AJ, Huang RR, Morton DL, Maecker HT: Immune responses in the draining lymph nodes against cancer: implications for immunotherapy. Cancer Metastasis Rev 2006, 25:233-242.

10. Zhao F, Dou J, Wang J, Chu L, Tang Q, Wang Y, Li Y, Cao M, Hu W, Hu K, Feng $\mathrm{He} X$, Gu N: Investigation on the anti-tumor efficacy by expression of GPI-anchored mIL-21 on the surface of B16F10 cells in C57BL/6 mice. Immunobiology 2010, 215:89-100

11. Zhao F, Dou J, He XF, Wang J, Chu L, Hu W, Yu F, Hu K, Wu Y, Gu N: Enhancing therapy of B16F10 melanoma efficacy through tumor vaccine expressing GPI-anchored IL-21 and secreting GM-CSF in mouse model. Vaccine 2010, 28:2846-2852.

12. Ulrike B, Schubert J, Wellner U, Schmalhofer O, Vincan E, Spaderna S, Brabletz T: A reciprocal repression between ZEB1 and members of the
miR-200 family promotes EMT and invasion in cancer cells. EMBO Rep 2008, 9:582-589.

13. Bracken $\mathrm{CP}$, Gregory PA, Kolesnikoff N: A double-negative feedback loop between ZEB1-SIP1 and the microRNA-200 family regulates epithelial- mesenchymal transition. Cancer Res 2008, 68:7846-7854

14. Chen D, Wang J, Zhang Y, Chen J, Yang C, Cao W, Zhang H, Liu Y, Dou J: Effect of down-regulated transcriptional repressor ZEB1 on the epithelial-mesenchymal transition of ovarian cancer cells. Int I Gynecol Cancer 2013, 23:1357-1366.

15. Chen D, Zhang Y, Wang J, Chen J, Yang C, Cai K, Wang X, Shi F, Dou J: MicroRNA-200c overexpression inhibits tumorigenicity and metastasis of $\mathrm{CD} 117^{+} \mathrm{CD} 44^{+}$ovarian cancer stem cells by regulating epithelial-mesenchymal transition. J Ovarian Res 2013, 6:50.

16. He X, Wang J, Zhao F, Yu F, Chen D, Cai K, Yang C, Chen J, Dou J: Antitumor efficacy of viable tumor vaccine modified by heterogenetic ESAT-6 antigen and cytokine IL-21 in melanomatous mouse. Immune Res 2012, 53:240-249.

17. Bendoraite A, Knouf EC, Garg KS: Regulation of miR-200 family microRNAs and ZEB transcription factors in ovarian cancer: Evidence supporting a mesothelial-to-epithelial transition. Gynecol Oncol 2010, 116:117-125.

18. Dou J, He XF, Cao WH, Zhao FS, Wang XY, Liu YR, Wang J: Overexpression of microRNA-200c in CD44+CD133+ CSCS inhibits the cellular migratory and invasion as well as tumorigenicity in mice. Cell Mol Biol 2013, 59:OL1861-OL1868.

19. Wang J, Zhou DD, He XF, Wang Y, Hu W, Jiang L, Dou J: Effect of downregulated $\beta$-catenin on cell proliferative activity, the sensitivity to chemotherapy drugs and tumorigenicity of ovarian cancer cells. Cell Mol Biol 2011, 57:OL1606-OL1613.

20. Zhang H, Wang J, Cai K, Jiang L, Zhou D, Yang C, Chen J, Chen D, Dou J: Downregulation of gene MDR1 by shRNA to reverse multidrug- resistance of ovarian cancer A2780 cells. J Cancer Res Ther 2012, 8:226-231.

21. Cittelly DM, Dimitrova I, Howe EN, Cochrane DR, Jean A, Spoelstra NS, Post MD, Lu X, Broaddus RR, Spillman MA, Richer JK: Restoration of miR-200c to ovarian cancer reduces tumor burden and increases sensitivity to paclitaxel. Mol Cancer Ther 2012, 11:2556-2565.

22. Liu SJ, Tetzlaff MT, Cui R, Xu X: miR-200c inhibits melanoma progression and drug resistance through down-regulation of Bmi-1. Am J Pathol 2012, 181:1823-1835.

23. Rosenberg SA, Sherry RM, Morton KE: Tumor progression can occur despite the induction of very high levels of self/tumor antigen-specific $\mathrm{CD}^{+} \mathrm{T}$ cells in patients with melanoma. J Immunol 2005, 175:6169-6176.

24. Dou J, Wu Y, Wang J, Zhao F, Chu L, Liu C, Wen P, Hu W, Hu K, He XF, Gu N: Eliciting protective immune responses against murine myeloma challenge in lymphopenia mice through adoptive transfer of tumor antigen-specific lymphocytes and immunization of tumor vaccine secreting mIL-21. Cancer Gene Ther 2010, 17:675-683.

25. Hervé L, Michèle F, Sylvie G: A novel flow cytometric assay for quantitation and multiparametric characterization of cell-mediated cytotoxicity. J Immunol Methods 2001, 253:177-187.

26. Yang $X$, Lin X, Zhong X, Kaur S, Li N, Liang S, Lassus H, Wang L, Katsaros D, Montone K, Zhao X, Zhang Y, Bützow R, Coukos G, Zhang L: Doublenegative feedback loop between reprogramming factor LIN28 and microRNA let-7 regulates aldehyde dehydrogenase 1-positive cancer stem cells. Cancer 2010, 70:9463-9472.

27. Jukic DM, Rao UN, Kelly L, Skaf JS, Drogowski LM, Kirkwood JM, Panelli MC Microrna profiling analysis of differences between the melanoma of young adults and older adults. J Transl Med 2010, 8:27.

28. Bierie B, Moses HL: Tumour microenvironment: TGF beta: the molecular Jekyll and Hyde of cancer. Nat Rev Cancer 2006, 6:506-520.

29. Massague' J: TGF in cancer. Cell 2008, 134:215-230.

30. Qu X, Shen L, Zheng Y, Cui Y, Feng Z, Liu F, Liu J: A signal transduction pathway from TGF- $\beta 1$ to SKP2 via Akt1 and c-Myc and its correlation with progression in human melanoma. J Invest Dermatol 2014, 134:159-167.

31. Thiery JP, Acloque H, Huang RY, Nieto MA: Epithelial-mesenchymal transitions in development and disease. Cell 2009, 139:871-890.

32. Liu S, Tetzlaff MT, Liu A, Liegl-Atzwanger B, Guo J, Xu X: Loss of microRNA-205 expression is associated with melanoma progression. Lab Invest 2012, 92:1084-1096.

33. Ulloa L, Doody J, Massagué J: Inhibition of transforming growth factor-beta/SMAD signalling by the interferon-gamma/STAT pathway. Nature 1999, 397:710-713. 
34. Hirano $T$, Satow R, Kato A, Tamura M, Murayama Y, Saya H, Kojima $H$, Nagano T, Okabe T, Fukami K: Identification of novel small compounds that restore E-cadherin expression and inhibit tumor cell motility and invasiveness. Biochem Pharmacol 2013, 86:1419-1429.

35. Wen FQ, Liu X, Kobayashi T, Abe S, Fang Q, Kohyama T, Ertl R, Terasaki Y, Manouilova L, Rennard SI: Interferon-gamma inhibits transforming growth factor-beta production in human airway epithelial cells by targeting Smads. Am J Respir Cell Mol Biol 2004, 30:816-822.

36. Weng H, Mertens PR, Gressner AM, Dooley S: IFN-gamma abrogates profibrogenic TGF-beta signaling in liver by targeting expression of inhibitory and receptor Smads. J Hepatol 2007, 46:295-303.

37. Kuga H, Morisaki T, Nakamura K, Onishi H, Noshiro H, Uchiyama A, Tanaka

M, Katano M: Interferon-gamma suppresses transforming growth factor-beta-induced invasion of gastric carcinoma cells through cross-talk of Smad pathway in a three-dimensional culture model. Oncogene 2003, 22:7838-7847.

doi:10.1186/1479-5876-12-68

Cite this article as: Wang et al: Regulation gene expression of miR200c and ZEB1 positively enhances effect of tumor vaccine B16F10/GPI-IL-21 on inhibition of melanoma growth and metastasis. Journal of Translational Medicine 2014 12:68.

\section{Submit your next manuscript to BioMed Central and take full advantage of:}

- Convenient online submission

- Thorough peer review

- No space constraints or color figure charges

- Immediate publication on acceptance

- Inclusion in PubMed, CAS, Scopus and Google Scholar

- Research which is freely available for redistribution 